\title{
Self-Assembled Masks for the Transfer of Nanometer-Scale Patterns into Surfaces: Characterization by AFM and LFM
}

2002

Vol. 2, No. 2

131-135

\author{
Maria-Victoria Meli, ${ }^{\dagger}$ Antonella Badia, ${ }^{\ddagger}$, Peter Grütter, ${ }^{\ddagger}$ and R. Bruce Lennox ${ }^{\star, \dagger}$ \\ Department of Chemistry, McGill University, 801 Sherbrooke Street West, \\ Montréal, Québec H3A 2K6, Canada, and Department of Physics, McGill University, \\ 3600 University Street, Montréal, Québec H3A 2T8, Canada
}

Received October 1, 2001; Revised Manuscript Received November 28, 2001

\begin{abstract}
A new methodology has been used to create an extensive array of nanometer-sized features. The $\mathrm{Ar}^{+}$milling of $\mathrm{Si} / \mathrm{SiO}_{x}$ and gold surfaces covered with a diblock copolymer mask with an intrinsic topological nanopattern yields transfer of the nanopattern. Mask removal was tracked using XPS, and the final arrays were characterized by AFM and LFM. Friction contrast observed throughout the transferred pattern is associated with a surface water layer.
\end{abstract}

The micromachining of materials with features in the 0.1 $\mathrm{nm}$ to $100 \mathrm{~nm}$ range $^{1}$ is of great interest for use in electronic devices, ${ }^{2}$ sensors (optical, pressure, electronic, etc.),${ }^{3-5}$ and separation science. ${ }^{6,7}$ Despite advances in lithography, it remains extremely difficult and expensive to realize nanometer-sized structures in an efficient and parallel process. Mask-based lithographic techniques, while being an entry into the parallel fabrication of features, face considerable challenges involving the interplay of the Rayleigh limit of the impinging etch beam $\left(h v, \mathrm{e}^{-}\right)$and the chemical demands of the mask. These problems are compounded by the difficulty in creating a mask with the desired nanometer features in the first instance. Given these challenges, we have explored the use of self-organizing polymer monolayers as a sacrificial etch mask. Building on our experience with the phenomenon of 2D self-ordered arrays of self-assembled diblock copolymers, ${ }^{8-14}$ we describe here a methodology in which a polymer film with an intrinsic topological pattern serves as a mask in the (nano)machining of $\mathrm{Si} / \mathrm{SiO}_{x}, \mathrm{SiO}_{2}$, and gold surfaces. The result is a high-fidelity transfer of the mask features into the underlying solid surface, generating an array of topologically distinct features that are ca. 50 $\mathrm{nm}$ in diameter, with a periodicity of $110 \mathrm{~nm}$, and exhibit friction contrast by lateral force microscopy (LFM).

There have been several successful demonstrations where

\footnotetext{
* Corresponding author. E-mail: bruce.lennox @mcgill.ca.

$\dagger$ Department of Chemistry.

Department of Physics.

$\S$ Present address: Department of Chemistry, Université de Montréal, C. P. 6128, succursale Centre-ville, Montréal, Québec, H3C 3J7, Canada.
}

pattern-forming materials have been used as masks for the creation of surface nanostructures. Möller and co-workers have shown that block copolymer reverse micelles deposited on a surface self-order and can be selectively derivatized to provide etch contrast. ${ }^{15,16}$ Register, Chaikin, and coworkers ${ }^{17-19}$ have been able to exploit the selective reactivity of one component of a block copolymer mask (i.e., polybutadiene domains in polystyrene matrices) to yield a versatile nanoscale mask system. Both these approaches rely on the mask having an intrinsic chemical pattern, whereupon follow-up chemical reactivity ${ }^{15-20}$ yields the desired topological features in the mask. One variation involves using a diblock copolymer that has intrinsic etch contrast. ${ }^{21}$ Transfer of these features then involves ion milling or reactive ion etching technologies. A more desirable configuration, which essentially eliminates a step in the fabrication process of the transferred pattern, is one where a mask with intrinsic topological contrast is used as a sacrificial mask in a chemically unselective etch process. Such a configuration was demonstrated by Clark and co-workers, where a 2D array of bacterial proteins was an effective topological mask toward an $\mathrm{Ar}^{+}$milling beam. ${ }^{22,23}$ In this case, $6 \mathrm{~nm}$ features with 25 $\mathrm{nm}$ periodicity resulted over $1 \mathrm{~cm}^{2}$ of surface. Several novel etch masks have recently been employed to create nanostructures in solid surfaces. For example, gold nanoparticles deposited on $\mathrm{Si}$ act as condensation nuclei and after etching lead to ca. $10 \mathrm{~nm}$ (diameter) by $100 \mathrm{~nm}$ (height) Si pillars. ${ }^{24}$ Rigid rod-like protein assemblies (microtubules) provide a mask, which, after etching, yields structures ca. $50 \mathrm{~nm}$ wide 


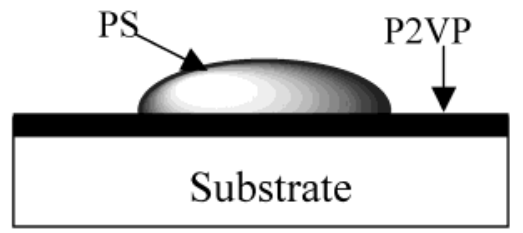

Figure 1. Schematic side profile of a PS-P2VP surface micelle. The PS core is ca. $4.5 \mathrm{~nm}$ high, and the $\mathrm{P} 2 \mathrm{VP}$ corona is ca. $1 \mathrm{~nm}$ thick. ${ }^{29}$

and several microns long. ${ }^{25}$ The successful introduction of topological contrast to a surface using a phase-separated polymer ${ }^{26}$ suggests that the block copolymer surface micelle mask could be used for pattern transfer in a manner similar to that used in the protein mask system.

Surface micelles formed from symmetric amphiphilic block copolymers at the air/water interface are circular in nature and have aggregation numbers in the range of 100125. ${ }^{9}$ The (narrow) polydispersity of the unimer combined with the distribution in aggregation numbers lead to the formation of an array of surface micelles with a narrow but finite size distribution. This surface micelle system has the unique design capability wherein the dimensions of the features in the desired mask can be tuned by choosing the appropriate block lengths. ${ }^{13,27}$

Langmuir-Blodgett (LB) monolayer films of surface micelles were prepared by evenly spreading $80-100 \mu \mathrm{L}$ of a $0.5 \mathrm{mg} / \mathrm{mL}$ chloroform solution of polystyrene(510)- $b$-poly2-vinylpyridine(480) $\left(M_{\mathrm{w}} / M_{\mathrm{n}}=1.06\right.$, Polymer Source Inc., Dorval, Canada) in ca. $5 \mu \mathrm{L}$ drops onto a $18 \mathrm{M} \Omega$ water surface $\left(23{ }^{\circ} \mathrm{C}\right.$, KSV 3000$)$. Twenty minutes elapsed after spreading before the Langmuir-Blodgett transfer began. The LB film was compressed at a rate of $5 \mathrm{~mm} / \mathrm{min}$ to a surface pressure of $2 \mathrm{mN} / \mathrm{m}$, and then transferred to a clean substrate $^{28}$ at $1 \mathrm{~mm} / \mathrm{min}$ (upward deposition). The resulting LB films consist of a quasi-hexagonal array of surface micelles composed of disk-like polystyrene (PS) cores surrounded by a continuum of poly-2-vinylpyridine (P2VP) coronae (Figure 1). ${ }^{8,11,29}$ The monolayer-coated substrate was then exposed to an $\mathrm{Ar}^{+}$ion beam at $5 \mathrm{keV}(I=70 \mathrm{nA}$, flux $=3.5 \mu \mathrm{A} / \mathrm{cm}^{2}, 50^{\circ}$ from surface normal) for a variety of exposure times (60 to $900 \mathrm{~s}$ ). The progress of the ionmilling process was tracked by X-ray photoelectron spectroscopy (XPS) with a VG ESCALAB 220i-XL spectrometer employing a monochromatic Al K $\alpha$ X-ray source $(h v=$ $1486.6 \mathrm{eV}$ ). The milled substrates were subsequently imaged using atomic force microscopy (AFM) and lateral force microscopy (LFM) with a Digital Instruments Multimode AFM (Nanoscope IIIA) using oxide-sharpened silicon nitride tips $(k=0.58 \mathrm{~N} / \mathrm{m}$, Digital Instruments).

Figure 2 depicts typical AFM images of the PS-P2VP surface micelle film transferred to a $\mathrm{Si} / \mathrm{SiO}_{x}$ substrate (Semiconductor Processing Inc., Ottawa, Canada). The surface micelle pattern extends over the entire substrate (several $\mathrm{cm}^{2}$ ). A typical $1 \mu \mathrm{m}^{2}$ area of the symmetric diblock $\left(\mathrm{PS}_{510}-\mathrm{P} 2 \mathrm{VP}_{480}\right)$ used here results in surface micelles whose dimensions are $4.5 \pm 0.5 \mathrm{~nm}$ (height), and $50 \pm 5 \mathrm{~nm}$ full width at half-maximum (fwhm), and which form a pattern with a periodicity of $110 \pm 10 \mathrm{~nm}$ (Figure 2). The range of

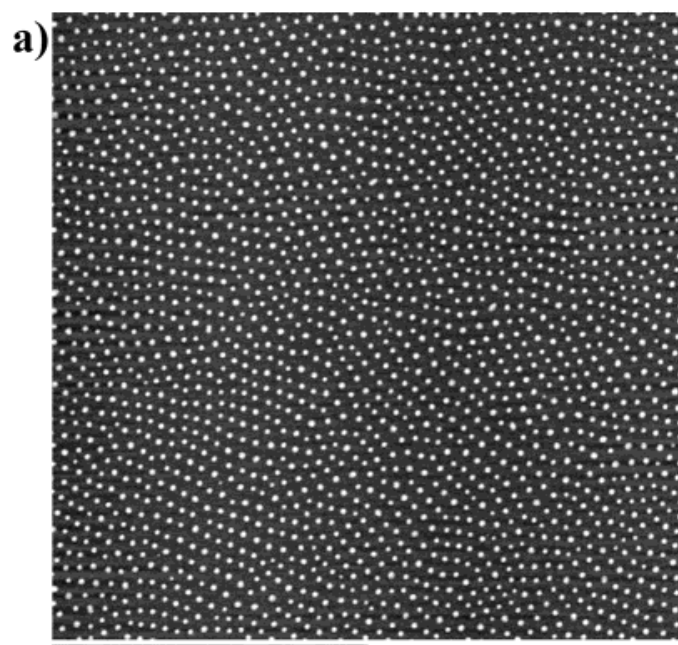

b)

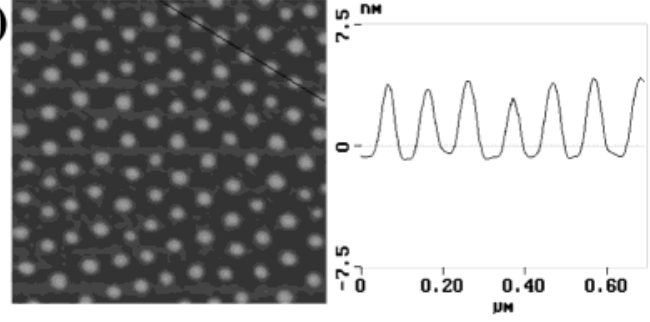

Figure 2. AFM images of a PS-P2VP surface micelle film deposited on $\mathrm{Si} / \mathrm{SiO}_{x}$ obtained with tapping mode imaging using a Si cantilever $\left(f_{\mathrm{o}} \sim 300 \mathrm{kHz}\right.$, NanoSensors). (a) $5 \mu \mathrm{m} \times 5 \mu \mathrm{m}$ area; (b) $1 \mu \mathrm{m} \times 1 \mu \mathrm{m}$ area (left) with cross-section profile (right).

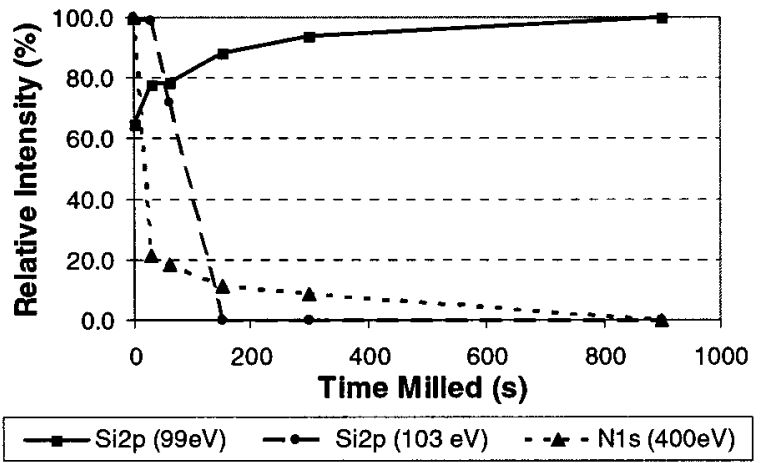

Figure 3. XPS relative intensities for the $\mathrm{Si} 2 \mathrm{p}$ and N1s maxima followed as a function of $\mathrm{Ar}^{+}$beam exposure time (0 to $900 \mathrm{~s}$ ). Lines serve only as visual aids.

these dimensions for a much larger area $\left(\sim 100 \mu \mathrm{m}^{2}\right)$ is however greater, where the average height is $4.5 \pm 1 \mathrm{~nm}$, the fwhm is $50 \pm 20 \mathrm{~nm}$, and the periodicity is $110 \pm 30$ nm.

Figure 3 shows the changes in relative intensity of the N1s and Si2p maxima obtained by XPS throughout the ionmilling process. Before ion milling begins, a prominent N1s peak associated with the P2VP block is observed at $400 \mathrm{eV}$. $300 \mathrm{~s}$ of ion milling leads to the almost complete disappearance of this peak to background noise levels, which is consistent with the polymer mask being removed. The Si2p peak $(99 \mathrm{eV})$, corresponding to elemental $\mathrm{Si}$, increases with longer $\mathrm{Ar}^{+}$beam exposure, while the peak at $103 \mathrm{eV}$ (corresponding to the native $\mathrm{Si}$ oxide layer) diminishes to the background noise level within $200 \mathrm{~s}$ of milling. In the 

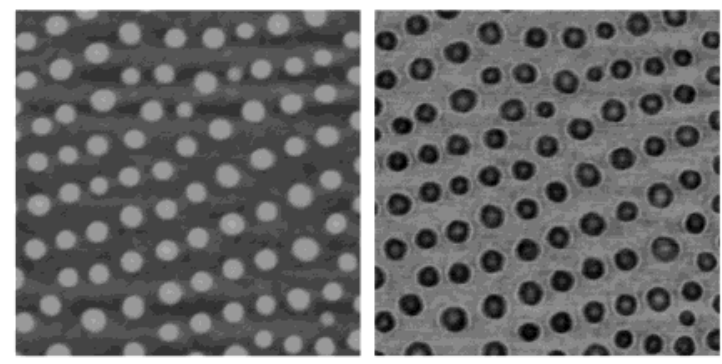

Figure 4. $1 \mu \mathrm{m} \times 1 \mu \mathrm{m}$ AFM (left) and LFM (right) images of PS-P2VP mask transferred into $\mathrm{Si} / \mathrm{SiO}_{x}$ after $300 \mathrm{~s}$ ion milling. Bright features in the AFM image correspond to higher height, and darker features in the LFM image correspond to lower friction. Note that the exact area imaged here is not the exact same area imaged in Figure $2 b$.

$150 \mathrm{~s}$ time frame the native oxide layer has been completely milled away, exposing underlying bulk Si.

AFM and LFM were performed in order to assess the topological and friction contrast which result from this masked etching process. Experiments were performed in ambient conditions (air, room temperature ca. $20^{\circ} \mathrm{C}$ ). All LFM images presented here were obtained by subtracting the retrace from the trace scan in order to essentially remove topography convolution. Figure 4 is a typical example of the results obtained for the pattern milled into a Si substrate. ${ }^{30}$ The AFM (topography) images suggest that the dimensions of the transferred pattern are not dependent on the milling time (60 to $900 \mathrm{~s}$ ). The contact mode AFM topological images clearly show that the pattern formed in the $\mathrm{Si} / \mathrm{SiO}_{x}$ surface is a high fidelity replica of the original diblock copolymer. Both the feature dimensions and periodicity of the mask are effectively transferred into the Si surface by the ion-milling process. The differences noted between Figure 2B and Figure 4 are within the limits of the feature size distribution of the original polymer mask (see Figure 4 caption). It is noteworthy that the chemically unselective ion milling process used yields feature heights in substrates that are similar to those of the mask. However, some variation in the heights of the features can occur if the substrate has a significantly different ion-milling rate than do the components of the mask.

LFM imaging yields an interesting contrast (Figures 4, 5, 6) that can arise from a number of sources. LFM contrast can arise from contact-related forces (adhesion, friction) as well as capillary effects ${ }^{31-41}$ associated with tip-adsorbed water interactions when imaging is performed under ambient conditions. The LFM contrast observed in Figures 4 and $5 b$ is related to the surface topography and not to chemical contrast on the surfaces. Two series of experiments were performed to help determine the origin of the contrast observed in the LFM image. First, nanopatterns were formed on both a $\mathrm{SiO}_{2}$ substrate (in the form of a $114 \mathrm{~nm}$ thick oxide layer on a $\mathrm{Si}$ wafer (AMC, Edmonton, Canada)) and a smooth $200 \mathrm{~nm}$ thick Au substrate. ${ }^{42-44}$ The observed LFM contrast (Figure 5) parallels that observed on the $\mathrm{Si} / \mathrm{SiO}_{x}$ substrate (Figure 4), suggesting that the difference in friction observed between the "hills" and the "valleys" in the nanopattern is not intrinsic to the substrate used. Instead,
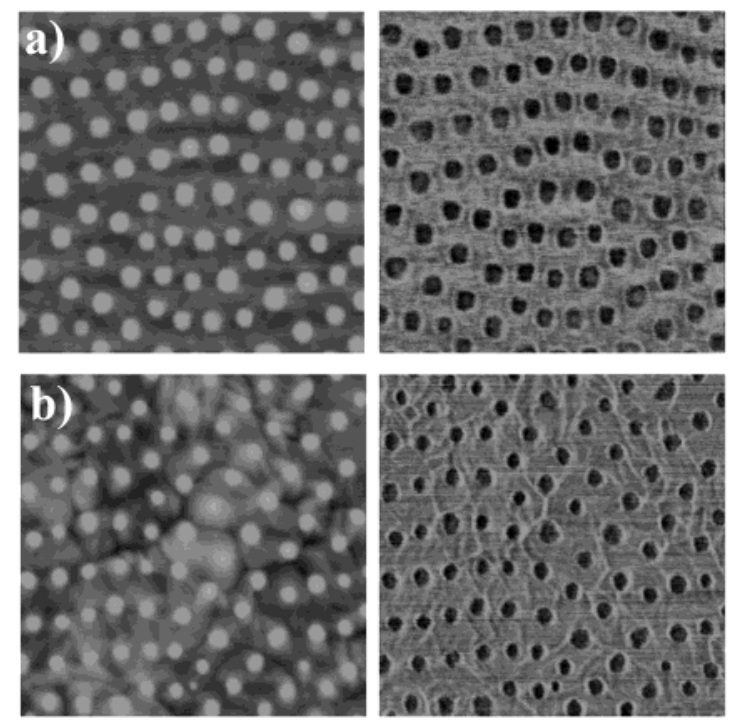

Figure 5. $1 \mu \mathrm{m} \times 1 \mu \mathrm{m}$ AFM (left) and LFM (right) images of PS-P2VP pattern milled for $150 \mathrm{~s}$ into (a) $\mathrm{Si} / \mathrm{SiO}_{2}$ and (b) $\mathrm{Au}$.

we find that the friction is related in some way to the intrinsic physical and/or chemical properties of the patterned surface. A second series of experiments was performed to establish whether the three regions of friction contrast (hill, valley, edge) are a feature of the nanoscale pattern or arise from another phenomenon. $\mathrm{Si} / \mathrm{SiO}_{x}, \mathrm{Si} / \mathrm{SiO}_{2}$, and $\mathrm{Au}$ substrates were exposed to similar ion-milling conditions as above (5 $\mathrm{keV}, 25 \mu \mathrm{A} / \mathrm{cm}^{2}, 50^{\circ}$ from surface normal, MET-ETCH, Gatan Inc.) but instead of the polymer mask, a Super2000 mesh $\mathrm{Cu}$ TEM grid $(7.5 \mu \mathrm{m}$ holes separated by $5 \mu \mathrm{m}$ bars, Canemco Inc., St. Laurent, Canada) was used as a mask. Typical AFM/LFM images of the resulting surfaces (Figure 6) reveal that the areas exposed to the ion-beam exhibit lower friction than do the masked areas. A variation of this experiment was also performed wherein the substrates were first patterned by ion milling through a TEM grid mask, the mask was removed, and the samples were reexposed to the ion-milling beam for another $60 \mathrm{~s}$. The substrates subjected to this sequential milling show the same LFM characteristics, establishing that increased ion-beam exposure leads to lower LFM-measured friction. The nanoscale roughness induced by the ion-milling process is believed to promote the formation of a thin water layer once the substrate is reexposed to ambient atmosphere. This water layer gives rise to a lesser friction than that of the unmilled regions. Moreover, the regions corresponding to the feature edges in both the nanoscale and microscale patterned $\mathrm{Si} / \mathrm{SiO}_{x}$ samples (Figures 4 and $6 \mathrm{a}$ ) have a pronounced lessening of the friction. ${ }^{45}$ These results suggest that lower friction is linked to the details of the topography (nanoscale roughness and curvature) of the surfaces.

LFM-measured friction is known to be exceptionally sensitive to the thickness of the adsorbed layer of water. For example, a $3 \AA$ thick layer of water has been distinguished by LFM on a mica surface as having lower friction than mica itself. ${ }^{31}$ It is thus very likely that the observed decrease in friction at the etch edge region arises because it has a thicker 


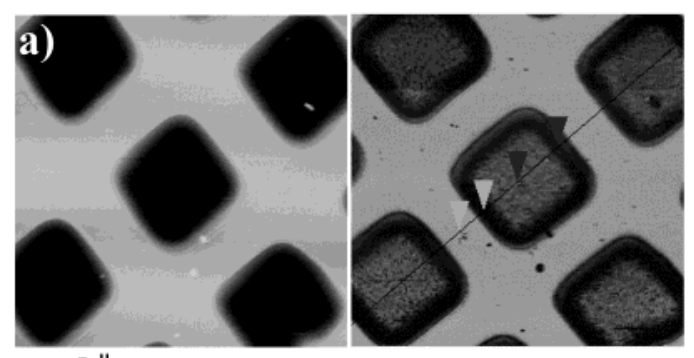

b) $\stackrel{\square}{-}$
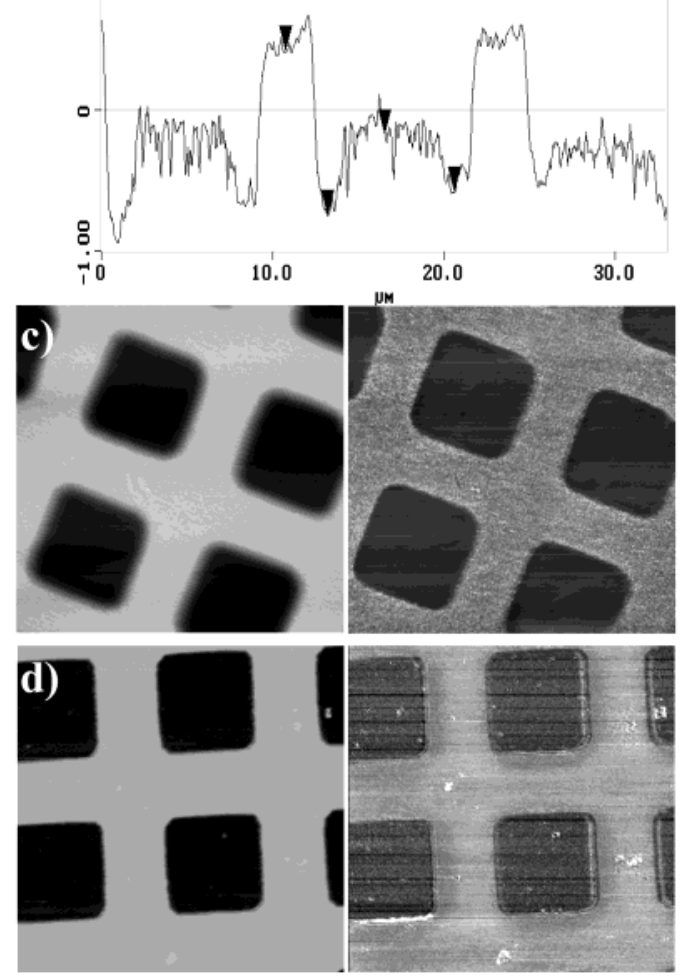

Figure 6. $25 \mu \mathrm{m} \times 25 \mu \mathrm{m}$ AFM (left) and LFM (right) images of substrates ion milled through a superfine mesh TEM grid. (a) 100 nm-deep pits formed by milling for $300 \mathrm{~s}$ into $\mathrm{Si} / \mathrm{SiO}_{x}$. (b) Crosssection profile for LFM image in (a). (c) $60 \mathrm{~nm}$-deep pits formed from milling for $150 \mathrm{~s}$ into $\mathrm{Si} / \mathrm{SiO}_{2}$. (d) $100 \mathrm{~nm}$-deep pits formed from milling for $60 \mathrm{~s}$ into $\mathrm{Au}$.

water coatingthan do the adjacent areas. However, it is also possible that the LFM experiment is generating the observed decrease in friction at the feature edges as a result of larger capillary forces between the tip and sample at the feature edges than at flat regions on the pattern. The possibility that water accumulates to a greater extent at the edges than elsewhere is intriguing, and we are currently investigating the relationships between substrate topologies, surface energy variations, and conformal vs nonconformal water layers on surfaces, as well as the effects of capillaries formed between the AFM tip and curved versus flat surfaces.

In summary, the self-assembly of a sacrificial mask with a topologically distinct array of nanometer-sized features has successfully been applied toward pattern transfer into smooth solid surfaces using a straightforward milling process. The high-fidelity pattern transfer process and removal of the mask were monitored using XPS, AFM, and LFM. Friction contrast observed in the nanopattern is a consequence of the topography introduced to the surfaces. Further work will concentrate on the investigation of nonconformal wetting and the adhesion of water onto the topological features, as well as the application of this pattern-transfer methodology to the generation of topologically and chemically distinct features in an array.

Acknowledgment. We thank NSERC Canada for financial support for this research. We also thank Georges Veilleux for assistance with the XPS experiments, Benjamin Smith for helpful discussions regarding the AFM/LFM experiments, and Miriam Rafailovich and Jason Li for discussions related to reference 26 .

\section{References}

(1) Kovacs, G. T. A.; Petersen, K.; Albin, M. Anal. Chem. 1996, 68, $407 \mathrm{~A}-412 \mathrm{~A}$

(2) Ogino, T.; Hibino, H.; Homma, Y.; Kobayashi, Y.; Prabhakaran, K.; Sumitomo, K.; Omi, H. Acc. Chem. Res. 1999, 32, 447-454.

(3) Lauks, I. Acc. Chem. Res. 1998, 31, 317-324.

(4) Semancik, S.; Cavicchi, R. Acc. Chem. Res. 1998, 31, 279-287.

(5) Berger, R.; Delamarche, E.; Lang, H. P.; Gerber, C.; Gimzewski, J. K.; Meyer, E.; Güntherodt, H.-J. Science 1997, 276, 2021-2024.

(6) Volkmuth, W. D.; Austin, R. H. Nature 1992, 358, 600-602.

(7) Figeys, D.; Pinto, D. Anal. Chem. 2000, 72, 330A-335A.

(8) Zhu, J.; Eisenberg, A.; Lennox, R. B. J. Am. Chem. Soc. 1991, 113, $5583-5588$.

(9) Zhu, J.; Lennox, R. B.; Eisenberg, A. Langmuir 1991, 7, 15791584.

(10) Zhu, J.; Hanley, S.; Eisenberg, A.; Lennox, R. B. Makromol. Chem. 1992, 53, 211-220.

(11) Cox, J. K.; Eisenberg, A.; Lennox, R. B. Curr. Opin. Colloid Interface Sci. 1999, 4, 52-59, and references therein.

(12) Zhu, J.; Lennox, R. B.; Eisenberg, A. J. Phys. Chem. 1992, 96, 47274730 .

(13) Zhu, J.; Eisenberg, A.; Lennox, R. B. Macromolecules 1992, 25, 6547-6555.

(14) Cox, J. K.; Yu, K.; Constantine, B.; Eisenberg, A.; Lennox, R. B. Langmuir 1999, 15, 7714-7718.

(15) Spatz, J. P.; Eibeck, P.; Mössmer, S.; Möller, M.; Herzog, T.; Ziemann, P. Adv. Mater. 1998, 10, 849-852.

(16) Spatz, J. P.; Herzog, T.; Mössmer, S.; Ziemann, P.; Möller, M. Adv. Mater. 1999, 11, 149-153.

(17) Park, M.; Harrison, C.; Chaikin, P. M.; Register, R. A.; Adamson, D. H. Science 1997, 276, 1401-1404.

(18) Li, R. R.; Dapkus, P. D.; Thompson, M. E.; Jeong, W. G.; Harrison, C.; Chaikin, P. M.; Register, R. A.; Adamson, D. H. Appl. Phys. Lett. 2000, 76, 1689-1691.

(19) Harrison, C.; Park, M.; Chaikin, P. M.; Register, R. A.; Adamson, D. H. J. Vac. Sci. Technol. B 1998, 16, 544-552.

(20) Thurn-Albrecht, T.; Steiner, R.; DeRouchey, J.; Stafford, C. M.; Huang, E.; Bal, M.; Tuominen, M.; Hawker, C. J.; Russell, T. P. Adv. Mater. 2000, 12, 787-791.

(21) Lammertink, R. G. H.; Hempenius, M. A.; van den Enk, J. E.; Chan, V. Z.-H.; Thomas, E. L.; Vancso, G. J. Adv. Mater. 2000, 12, 98103.

(22) Douglas, K.; Devaud, G.; Clark, N. A. Science 1992, 257, 642644.

(23) Holland, B. W.; Douglas, K.; Clark, N. A. Mater. Res. Soc. Symp. Proc. 1994, 330 (Biomolecular Materials by Design), 121-126.

(24) Tada, T.; Hamoudi, A.; Kanayama, T.; Koga, K. Appl. Phys. Lett. 1997, 70, 2538-2540.

(25) Fritzsche, W.; Böhm, K. J.; Unger, E.; Köhler, J. M. Appl. Phys. Lett. 1999, 75, 2854-2856.

(26) Zhu, S.; Gambino, R. J.; Rafailovich, M. H.; Sokolov, J.; Schwarz, S. A.; Gomez, R. D. IEEE Transactions on Magnetics 1997, 33 , 3022-3024.

(27) Zhu, J.; Eisenberg, A.; Lennox, R. B. Macromolecules 1992, 25, 6556-6562.

(28) Silicon substrates were cleaned by soaking for $5 \mathrm{~min}$ in boiling chloroform (Fisher Scientific, Canada, HPLC Grade)/ethanol 
(Anhydrous, Commercial Alcohols Inc., Canada) (50:50 v/v) solution, followed by ultrasonication for $2 \mathrm{~min}$.

(29) Li, Z.; Zhao, W.; Quinn, J.; Rafailovich, M. H.; Sokolov, J.; Lennox, R. B.; Eisenberg, A.; Wu, X. Z.; Kim, M. W.; Sinha, S. K.; Tolan, M. Langmuir 1995, 11, 4785-4792.

(30) AFM and LFM imaging was performed with a large range of applied loads ( $\sim 20$ to $200 \mathrm{nN}$ approximately) using oxide-sharpened silicon nitride tips (NanoSensors) with a variety of force constants $(k=0.06$, $0.12,0.32,0.56 \mathrm{~N} / \mathrm{m})$, and at a scan rate of $1 \mathrm{~Hz}$. LFM contrast was observed for the nanopatterned samples (60 to $900 \mathrm{~s}$ milling) when the load was kept low (less than approximately $80 \mathrm{nN}$ ). However, the contrast was (infrequently) observed to reverse such that areas that normally appeared to cause less lateral deflection, appeared to cause more lateral deflection. The cause of this contrast reversal is unclear; however, we suspect that it is related to changes in the ambient relative humidity (typically $\sim 60 \%$ relative humidity). Piner et al. ${ }^{31}$ have reported similar humidity effects on the LFM experiment when performed in air.

(31) Piner, R. D.; Mirkin, C. A. Langmuir 1997, 13, 6864-6868.

(32) Thundat, T.; Zheng, X. Y.; Chen, G. Y.; Warmack, R. J. Surf. Sci. 1993, 294, L939-L943.

(33) Binggeli, M.; Mate, C. M. Appl. Phys. Lett. 1994, 64, 415-417.

(34) Binggeli, M.; Mate, C. M. J. Vac. Sci. Technol. B 1995, 13, $1312-$ 1315 .

(35) Sugimura, H.; Okiguchi, K.; Nakagiri, M.; Miyashita, M. J. Vac. Sci. Technol. B 1996, 14, 4140-4143.

(36) Crassous, J.; Charlaix, E.; Loubet, J. Phys. Rev. Lett. 1997, 78, 24252428.
(37) Hu, J.; Xiao, X.-D.; Ogletree, D. F.; Salmeron, M. Surf. Sci. 1995, 327, 358-370

(38) Sedin, D. L.; Rowlen, K. L. Anal. Chem. 2000, 72, 2183-2189.

(39) Quon, R. A.; Ulman, A.; Vanderlick, T. K. Langmuir 2000, 16, 8912-8916.

(40) Thundat, T.; Zheng, X.-Y.; Chen, G. Y.; Sharp, S. L.; Warmack, R. J.; Schowalter, L. J. Appl. Phys. Lett. 1993, 63, 2150-2152.

(41) Fujihira, M.; Aoki, D.; Okabe, Y.; Takano, H.; Hokari, H. Chem. Lett. 1996, 7, 499-500.

(42) $\mathrm{SiO}_{2}$ and $\mathrm{Au}$ nanopatterns were also prepared under a variety of milling times (60 to 300 s), and the AFM and LFM images presented are consistent for all samples prepared within this range of ion-beam exposure. XPS was used to verify that the $\mathrm{SiO}_{2}$ and $\mathrm{Au}$ layers (114 and $200 \mathrm{~nm}$ respectively) had not been milled through.

(43) The smooth Au films were prepared by first evaporating $200 \mathrm{~nm} \mathrm{Au}$ onto a clean $\mathrm{Si}$ wafer at a rate of $2 \AA / \mathrm{s}$ at $5 \times 10^{-6}$ Torr. The $\mathrm{Au}$ surface was fixed to a glass slide with epoxy glue, and then pulled off the Si wafer, thus exposing a smooth surface of Au. This method is a variation of that described by Stamou et al. (ref 44).

(44) Stamou, D.; Gourdon, D.; Liley, M.; Burnham, N. A.; Kulik, A.; Vogel, H.; Duschl, C. Langmuir 1997, 13, 2425-2428.

(45) The friction contrast observed at the edges of the features was only observed for the $\mathrm{Si} / \mathrm{SiO}_{x}$ samples and has not been observed in the $\mathrm{Si} / \mathrm{SiO}_{2}$ or $\mathrm{Au}$ samples under the experimental conditions explored. Furthermore, UV/ozone cleaned and oxide-sharpened AFM tips are required to resolve these edge effects.

NL015639V 